\title{
6. Mechanical Specializations of Insect Ears
}

James F.C. Windmill*1, and Joseph C. Jackson ${ }^{2}$

${ }^{1}$ Centre for Ultrasonic Engineering

Department of Electronic \& Electrical Engineering

University of Strathclyde

204 George Street

Glasgow

G1 1XW

United Kingdom

james.windmill@strath.ac.uk

*corresponding author

${ }^{2}$ Centre for Ultrasonic Engineering

Department of Electronic \& Electrical Engineering

University of Strathclyde

204 George Street

Glasgow

G1 1XW

United Kingdom

joseph.jackson@strath.ac.uk

Keywords:

Active hearing; Antennal ears; Cicada ear; Directional hearing; Hearing mechanisms; Insect hearing systems; Locust ear; Mosquito ear; Moth ear; Tympanal ears; 


\begin{abstract}
In this chapter some of the mechanical specializations that insects have evolved to carry out acoustic sensory tasks are reviewed. Although it is easy to perceive insect hearing organs as simplistic compared to other animals, the mechanisms involved can be complex. This chapter therefore acts as an introduction to the complexities of some insect hearing systems, as viewed from a mechanical perspective. The chapter provides some of the background knowledge readers require to investigate the subject in greater depth, while acknowledging that this subject is an active, developing, and broad area of research. Following a brief background section on the physics of sound as applied to the insect ear, the mechanical function of several insect hearing organs is discussed in relation to the different acoustic parameters that different insect species need to evaluate, such as frequency, origin, and amplitude. A further section then follows to discuss the mechanical basis of active hearing, whereby energy is added to the hearing system to condition its acoustic response, again using available examples. Finally, the chapter concludes with a discussion on the current state-ofthe-art in this active research area, and makes some suggestions as to where the future may lead insect hearing mechanism researchers.
\end{abstract}

\title{
6.1 Introduction
}

The sense of hearing has evolved multiple times in the insects, fulfilling a variety of different tasks including communication (Greenfield, Chap. 2; Balakrishnan, Chap. 3) and defense (Pollack, Chap. 4). In this chapter some of the mechanical specializations that insects have evolved to carry out such acoustic sensory tasks are reviewed. Despite their perceived simplicity, the mechanics of the hearing organs of insects are complex. This chapter seeks only to introduce some of these systems, as seen from a mechanical perspective, providing the background knowledge required to delve further into the subject, which is an actively 
developing area of research. However, before considering the different mechanisms that insects use to sense sound it is important to understand the physical forces that their hearing organs are interacting with. So the initial question must be what is sound? What is it that the insects are trying to sense?

\subsubsection{What is Sound?}

Sound can be defined as any form of vibration through a medium, whether that is air, water or a solid. This vibration causes the medium's particles to be displaced temporarily. This displacement affects the surrounding particles such that they are displaced, resulting in a wave of particle displacements which is recognized as sound. The sound waves that insects are typically considered to hear are longitudinal, such that the particle displacement is parallel to the direction of travel of the wave, as shown in Figure 6.1. As sound is a wave, frequency (f), wavelength ( $\lambda$ ) and speed (c) are used to describe it through the following equation:

$$
c=f \lambda
$$

These are important characteristics when considering the sense of hearing in the insects. For a small animal like an insect the wavelengths involved in hearing can mean that evolution has led to interesting and complex mechanical solutions, some of which will be described in this chapter. 


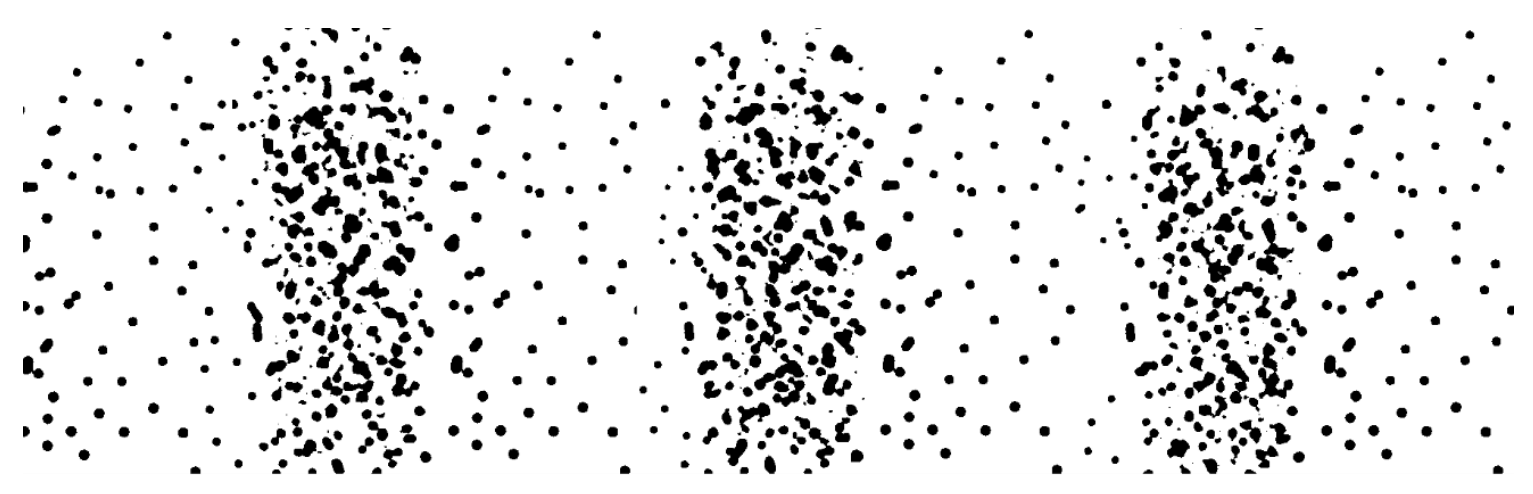

\section{Compression}

Rarefaction

Figure 6.1. Longitudinal sound waves traveling through a medium. These are sometimes also called compression waves, because they produce compression (the particles in the wave press closer) and rarefaction (the particles in the wave thin out). (C James Windmill.

\subsubsection{Sound Pressure and Particle Velocity}

One of the standard measures of the 'loudness' of a sound is the Sound Pressure Level (SPL). The change in pressure is caused by the compression and rarefaction of the air particles creating the sound wave. As these particles are in motion they have their own velocity that should not be confused with the speed of the sound wave moving through the medium. The unit used is Pascal (Pa), as Newtons per square meter. However, as the sensed change in pressure may be $10^{6}$ (i.e. from $\mu \mathrm{Pa}$ to $\mathrm{Pa}$ ) sound pressure level is typically quoted in decibels, $(\mathrm{dB})$, as the change relative to a reference sound pressure (i.e. $20 \mu \mathrm{Pa}$ ). In water this reference level is normally $1 \mu \mathrm{Pa}$, however the acoustic impedance of water is different to that of air, which means that direct comparisons between sound in air and water are not as simple as changing the $\mathrm{dB}$ reference calculation. Typically a sound pressure level measurement is quoted for a distance from a sound source, usually one meter, but it can be any distance chosen.

Finally, confusion is often caused by the use of sound level to mean sound pressure level or sound intensity level. Sound intensity is a measure of the sound power per unit area, 
given as Watts per square meter. Intensity can be considered a sound energy quantity, which is not the same as pressure (a force quantity). Therefore it is incorrect to say intensity when discussing pressure level. The convention to use when talking about sound pressure level is therefore to say the amplitude of sound measured. However, it should be noted that the normal reference levels of $20 \mu \mathrm{Pa}$ for sound pressure, and $1 \mathrm{pW} / \mathrm{m}^{2}$ for sound intensity, mean that in $\mathrm{dB}$ the sound pressure and intensity are calculated to be the same number, although they are different physical measurements.

\subsubsection{Sound Attenuation}

Sound attenuates as it travels through a medium. However the extent of the attenuation depends on a number of factors. The attenuation is proportional to the distance the sound travels, and also the viscosity of the medium that the sound travels through. Other factors include whether the medium itself is traveling, for example in air movements in windy conditions, and also the density and pressure of the medium (Bennet-Clark, 1998). There is also an added dissipative effect that causes further attenuation relating to the frequency of the sound. The predominant mechanism of such attenuation is proportional to the square of frequency, so at relatively low frequencies (e.g. $1 \mathrm{kHz}$ ) this effect is approximately $0.005 \mathrm{~dB}$ per meter (assuming air temperature of $20^{\circ} \mathrm{C}$ and relative humidity at $50 \%$ ). As frequency increases this attenuation factor becomes far more important, such that at $100 \mathrm{kHz}$ it is $3.28 \mathrm{~dB}$ per meter.

\subsubsection{Sound over Distance: Near field and Far field sounds}

The sound field in a free field is typically divided into two regions by distance; the near field and the far field. The near field is also often subdivided into two regions, for example the hydrodynamic and geometric near field (Bies \& Hansen, 2009). However, in the 
context of insect hearing systems this section will discuss only the near and far field. Readers interested in delving deeper into this subject are recommended to read Bies and Hansen, 2009, or other appropriate physical acoustics books. It is also the case that the discussion of near and far fields can relate to either the distance from a source or from a receiver. This chapter reviews the mechanics of receivers, the insect ears, and so the definition of near and far field relates to the distance from the sound source that the insects are hearing.

The near field is the region immediately adjacent to the vibrating surface of the source, extending outward a distance much less than one wavelength. This region is characterized by fluid motion that is not directly associated with sound propagation, and where the acoustic pressure is out of phase with the local particle velocity in the medium. The region of the sound field extending beyond the near field towards infinity is the far field, where, in the absence of reflecting surfaces, sound pressure levels attentuate at the rate of 6 $\mathrm{dB}$ for each doubling of distance (not including viscous losses). The far field is characterized by satisfying the following criteria,

$$
r » \lambda /(2 \pi), \quad r » l, \quad r » \pi l^{2} /(2 \lambda)
$$

where $r$ is the distance from the source to the measurement position, $\lambda$ is the wavelength of the sound and $l$ is the characteristic source dimension. In many cases the boundary between the near and far field is given as approximately $\lambda / 6$. This is simply an approximation, where 6 is taken for $2 \pi$.

\subsubsection{Introducing Insect Ear Mechanics}

As mentioned before, sound has both pressure and particle velocity components, and therefore it is possible to preferentially detect one or the other. As both apply forces in different ways, the sensor used to detect pressure is fundamentally different to the sensor required for particle velocity detection (see Fig. 6.2). The attenuation of sound pressure with 
distance is less than that of particle velocity $\left(1 / \mathrm{r}\right.$ vs. $\left.1 / \mathrm{r}^{2}\right)$, meaning that over long distances, pressure is more detectable.

As the pressure component travels farthest, it is therefore the choice for long-range communication in land animals. To detect the pressure component, it is necessary to build a sensor that moves in response to changes in pressure. The biological solution is the tympanum (or tympanal membrane), a thin membrane of cuticle stretched over a chamber filled with air (or sometimes fluid). Tiny changes in pressure due to sound causes the tympanum to feel a force proportional to the pressure difference across the membrane, which in turn causes a deflection of the tympanum. As the pressure is oscillatory, so is the motion of the tympanum. Attached to a tympanum (not necessarily directly) is a mechanosensory system that is stretched and compressed by this tympanal motion, converting this into electrical signals in nerve cells. This solution to sound detection is used by many animals, particularly in insects and the ear drum of the vertebrate ear. In many cases it is the only mechanical stage in sound transduction before the neurons, but in more complex systems it can be the first part of a long chain of energy transduction. Tympanal sensors are very sensitive, typically detecting sub-nanometer deflections of the attachment site of the mechanosensory neurons.

Detecting the particle velocity component of sound requires a different method. A sensor must be driven by being viscously dragged by the particle motion. These sensors are either hairs or antennae, and are ubiquitous in insect and arthropod taxa. A major problem with this type of sensor is that it must be external, projecting away from the body to allow efficient sampling of the fluid flow. This renders them liable to be damaged as they cannot be protected from the environment. However, these external antennae can be multi-functional, with multiple sensors giving the ability to detect sound, vibration, wind speed, and also perform chemo- and thermo-reception. They are also very sensitive so that, for example a 
mosquito can detect antennal displacements of $\pm 7 \mathrm{~nm}$, corresponding to sound particle displacements of $\pm 1 \mathrm{~nm}$ (Göpfert \& Robert, 2001).

A

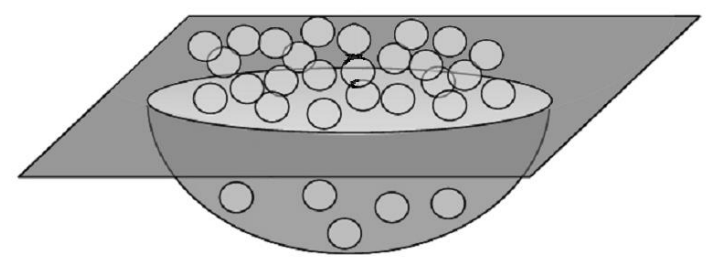

B

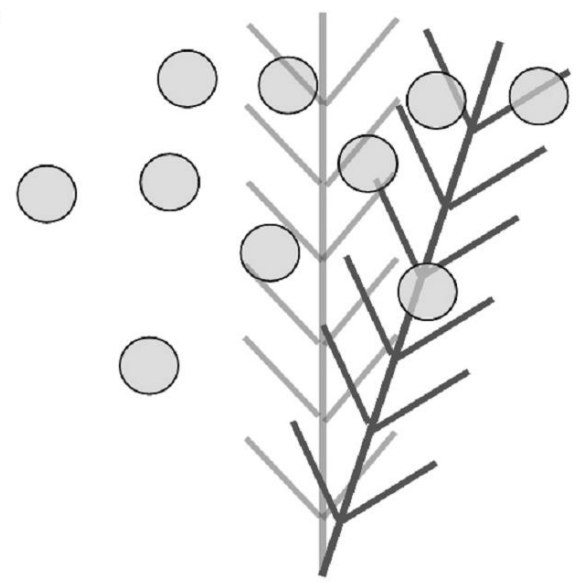

Figure 6.2. Particles interacting with acoustic structures. A. The tympanum separates regions of normal air pressure and sound-induced changes in pressure such that the mismatch in pressure creates a force that deforms the membrane. B. The antenna experiences a force caused by the viscous drag of the particle motion of sound. @ Joseph Jackson.

When some of the literature on the ears of insects is examined, different insect ears are often described as near-field or far-field detectors, as well as particle velocity or pressure detectors. The interchangeable use of these terms can be confusing, so it is worth considering how they relate. Firstly it should be clear that when discussing near and far field this relates to the distance the ear is from a sound source, not the size of the ear compared to the sound wavelength. It is therefore appropriate to consider what frequency, and so wavelength, of sound different ear structures are attempting to detect. Low frequencies have long wavelengths, which also means the near field of such sounds extends further in distance. Within the near field, the particle velocity is high, so particle velocity sensors have typically evolved for detection in the near field of low frequency sound sources. For example, 
mosquitoes and flies listen for the low frequency $(100-400 \mathrm{~Hz})$ flight tones of their own species for mating. They can utilize a particle velocity ear for detecting sound in the nearfield (some 10's of centimeters), where the particle velocity is greatest. Locusts and moths hear higher frequencies, into ultrasound (> $20 \mathrm{kHz})$, where the near field is a few millimeters. To hear sounds of an approaching predatory bat, at a useful distance (as the bat flies at several meters per second), particle velocity is significantly attenuated, so the preferential hearing system to use is a pressure detection system, which is thus considered a far field sensor. That is not to say that particle velocity in the far field is not there to be detected, it is simply that it has attenuated so much that the pressure measurement is far more appropriate.

\subsubsection{Acoustic Impedance and Impedance Matching}

Acoustic impedance is the ratio of complex acoustic pressure to complex acoustic volume velocity. It can therefore be considered as the complex representation of the acoustic resistance of a medium. As such acoustic impedance can be thought of as the opposition by a medium to the flow of energy. It is conventionally given the denotation $\mathrm{Z}$, and measured in Pa. $\mathrm{m}^{-3} . \mathrm{s}$ or in Rayl. $\mathrm{m}^{-2}$, i.e. 1 Rayl is $1 \mathrm{~kg} \cdot \mathrm{s}^{-1} \cdot \mathrm{m}^{-2}$. It is a ratio of complex numbers because, as the use of the word impedance implies, it is a combination of resistance (energy dissipation) and reactance (energy conservation). In the context of insect hearing systems this chapter will only discuss the consequences of acoustic impedance. For detailed treatments of this subject readers are recommended to consider appropriate physical acoustics books (Fletcher, 1992).

The concept of acoustic impedance is important when considering insect ears as it directly influences the mechanisms of the hearing system. In order for a sensor, the ear, to work most efficiently its structure must match the impedance of the medium through which the sound travels. If the impedance is not closely matched then the energy within the medium will not be transferred into the ear structure (causing it to move), but instead it would be 
reflected. A good sensor (ear) would have to be light and viscous to be susceptible to the motion of air particles or the minute pressure differences caused by sound. In fact, a structure with similar physical properties to the medium surrounding it would therefore be the perfect sensor, as this involves the most efficient transfer of energy from medium to ear. Taking the example of the insect tympanal membrane, this is a structure which is extremely thin (light), which vibrates in response to changes in pressure between the external and internal sides of the membrane. Therefore the membrane vibrates most efficiently when the acoustic impedance of the sound medium is matched on each side. This impedance matching is achieved by creating an air space directly behind the membrane through a modification of the insect's internal, tubular tracheal system. There are variations and exceptions to this rule, for example the green lacewing (Chrysopa carnea) is reported to have a fluid chamber backing its tympanal membrane (Miller, 1970).

Finally, the specific acoustic impedance of a sound-carrying medium is dependent on the physical properties of that medium. For example, the density of water is $\sim 1000 \mathrm{~kg} / \mathrm{m}^{3}$, whereas air's density is approximately 800 times less, depending on temperature and altitude. Likewise the speed of sound in water is $\sim 1500 \mathrm{~m} / \mathrm{s}$; over 4 times greater than that of air. Therefore the (specific) acoustic impedance of water is approximately 3,500 times higher than that of air. This difference means that a sound of equal pressure in water to that of a sound in air is actually 3,500 times less intense than the sound in air. This is because in the air, with its lower acoustic impedance, sound has a much greater particle velocity and displacement amplitude than water. It is this variation that leads to important differences when considering acoustics in air and underwater acoustics (see section 1.2). 


\subsubsection{Pressure Difference and Pressure Gradient}

Two terms that are often used to describe both microphones and tympanal insect ears are pressure difference (or sometimes pressure operated) and pressure gradient. Microphones and tympanal insect ears both work by sensing the pressure difference on either side of a thin sheet, most often called a diaphragm for microphones, and a membrane for insect ears, although the terms are sometimes interchanged.

In a pressure difference microphone, one side of the diaphragm is open to the atmosphere and is able to respond to the microscopic changes in pressure representing sound. The other side faces an enclosed volume which effectively contains a fixed "reference" air pressure. This means that the diaphragm moves in response to the difference between the pressure of the passing sound wave and the reference pressure within it. This is how an insect tympanal ear is conventionally imagined to function. However, in insects the air chamber behind the tympanal membrane is not completely enclosed, as the chamber is part of the tracheal system of air tubes used by insects to breathe. This means that the use of this nomenclature for the insects is actually dependent on the frequency of the sound.

In a pressure gradient microphone the diaphragm is still sensitive to the difference in sound pressure on either side; however, both sides are exposed to the (same) atmosphere, and therefore to the changing pressure caused by passing sound waves. In the microphone, if a sound wave arrives in the plane of the diaphragm then there are identical pressures on both sides and so no movement of the diaphragm. There is no pressure gradient across the diaphragm and so the microphone cannot detect sounds in this orientation. If sound arrives perpendicular to the diaphragm it will create a large pressure difference between front and rear, and it will be moved a maximum amount as a result. In insects, if the frequency of the sound arriving at the body is very low, and as such, the wavelength is much greater than the body size of the insect. It is then possible to imagine that the tympanal membrane may 
undergo a pressure change on both sides, as the pressure change appears through the tracheal system. Thus, the force is exerted both externally on the tympanal membrane due to a primary sound pressure contribution from the outside, and also via a secondary, attenuated contribution from the inside of the membrane. As such, depending on frequency, insect tympanal ears can be described as either pressure difference or pressure gradient. However, within the literature the reader will often find these terms used interchangeably, and therefore incorrectly.

\subsection{The Passive Mechanics of Insect Ears}

Insect auditory organs have previously been described to have four main types; trichoid sensilla, Johnston's organs, subgenual organs, and tympanal organs (Yack, 2004). Subgenual organs detect substrate-borne vibrations, and are discussed in Chapter 8 . Of the three remaining types, the trichoid sensilla and the Johnston's organ (in combination with the insect's antenna) are used to detect sound particle velocity, and the tympanal organs to detect sound pressure (see Fig. 6.2). Trichoid (filiform) sensilla are hair-like cuticular projections, while the Johnston's organ sits within the pedicel of the insect's antenna. The detection of particle velocity $\left(\mathrm{v}_{\mathrm{p}}\right)$ is achieved through drag on the filiform or antenna, as the antenna or sensilla experiences a force $\mathrm{F}=\mathrm{bv}_{\mathrm{p}}$ where $\mathrm{b}$ is the viscous drag of the antenna or sensilla. This force causes the deflection of the antenna or sensilla, stimulating the sensory systems at their base. The tympanum acts to separate regions of normal air pressure $\mathrm{P}_{0}$ and soundinduced changes in pressure $P$. This mismatch in pressure creates a force $F=A \Delta P$, where $A$ is the area of the membrane. Deformation of the membrane caused by this force is detected by the sensory neuron structure attached either directly, or through a secondary mechanism, to the membrane. 
It should also be recognized that there is a massive shift in the magnitudes that are under consideration when investigating insect ear mechanisms. The diameter of a tympanal membrane may be measured in hundreds of micrometers, if not millimeters. The thickness of that membrane will typically be measured from tens of micrometers down to less than one micrometer. However, in response to sound levels at which the ear is neurally sensitive, the tympanal membrane displacement is typically measured in nanometers, from tens of nanometers down to a fraction of a nanometer. This is a magnitude difference in meters of approximately ten to the power six. Anyone approaching this research field must keep this in mind, especially as figures in papers are always produced to show the data such that it is easy to view. It is easy to miss the consideration of scale.

In this section some of the passive mechanisms by which insect ears interact with incident sounds in order to undertake an initial stage of information processing are reviewed. Passive refers to the fact that these interactions require no additional energy to be imparted into the hearing system; they are simply mechanical responses of the hearing structures to sound. The examples reviewed have been grouped into sound frequency analysis and sound direction analysis. This is a purely arbitrary decision made in order to bring some structure to the section.

\subsubsection{Frequency Tuning}

\subsubsection{Moth}

All moths can be described as having the 'simplest' possible insect ear morphology. This is because the ears of moths have a very low number of sensory neuron structures directly attached to the tympanal membrane. It cannot therefore passively distinguish different frequencies, other than through the fact that the mechanical tuning of its ear membrane will make it preferentially sensitivity to a certain frequency bandwidth, as the 
neuronal structures are not mechanically tuned within that bandwidth. The earliest studies of the moth ear go back to the work of Roeder in the 1950s (e.g. Roeder, 1957), although many researchers have followed since.

Hearing in moths has evolved independently at least five times. In the Noctuidea the ears are found in the metathoracic segment, with two sensory neurons directly attached to the tympanal membrane (although Notodontidae have only one neuron). In Pyraloidea, Geometroidea and Drepanoidea the ears have four neurons, and are located in the abdomen. Finally, those moths in the Sphingidae that hear have ears on the proboscis. The principal reason for the evolution of hearing in moths is to detect the ultrasonic echolocation calls of predatory bats. Therefore, moth ear frequency sensitivity is comparable with typical bat echolocation frequencies, of about 20 to $60 \mathrm{kHz}$.

Although the location, and to some extent morphology, of the ears found in the different hearing moth species is diverse, the conceptual understanding of the moth ear as a passive mechanical receiver is common across species (see section 6.3.2 for discussion of active mechanisms in insect hearing). The general structure of the moth ear can be pictured as a tympanal membrane that is approximately circular in nature, with a single sensory neuron attachment site near the membrane's center point, as demonstrated in Figure 6.3. Thus, as a passive hearing structure the moth ear can be thought of as a simple resonant membrane. Therefore the frequency tuning of a moth ear should directly scale to the size of the tympanal membrane. In general this is the case, with a direct correlation between size and frequency, such that larger moths are tuned to lower frequencies, and smaller ones to higher frequencies. 


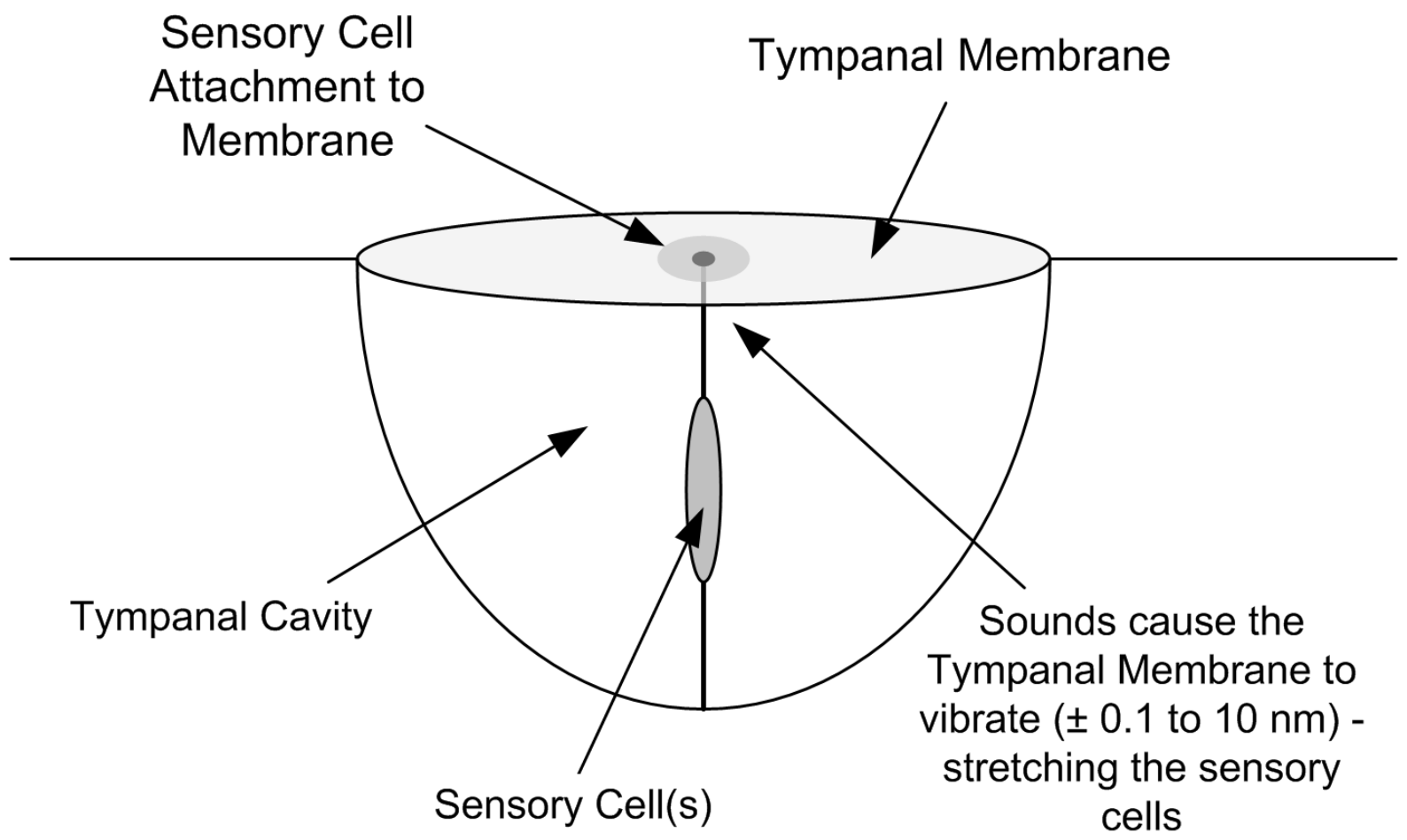

Figure 6.3. Schematic of the structure of a moth ear - as the 'simplest' insect ear. (C) James Windmill.

Taking the assumption that different sized moth ears will have very similar material properties, then the difference in tympanal membrane size also has an effect on amplitude sensitivity. A larger membrane is subject to a greater overall force compared to a smaller one when the same sound pressure is applied (assuming each membrane is the same thickness). This means that the neuron attachment site of the larger membrane will be deformed by a greater amount for that same sound pressure. This correlation between moth size and ear sensitivity is clearly seen when the neural sensitivity of different moths is considered (Surlykke, 1999). This increased sensitivity means that the larger moth, which would be more noticeable to a hunting bat, has the advantage of being able to detect the bat's echolocation calls from a greater distance than a smaller moth. Thus the apparent liability of greater size is offset by the increase in hearing sensitivity. 
Of course, while it is convenient to imagine the moth ear as a simple resonant membrane, the actual morphology is more complex. For example, the noctuid tympanal ear has two membranes, the tympanal membrane and an adjacent conjunctivum membrane (also known as the counter-tympanic membrane, depending on the species). The tympanal membrane is thin, typically transparent, with a thicker area near the center where the neural attachment is sited. The second adjacent membrane is much thicker, and has no neural attachment. A thicker ridge of cuticle runs between the two membranes. The mechanical response of the thinner membrane is not that of a simple membrane mode. Rather, the attachment point moves far more than the thin membrane surrounding it. Also, the two membranes deflect in anti-phase at low frequencies. Then as frequency increases, the deflection of the thicker secondary membrane reduces until it appears to remain stationary. At this point no function related to the mechanics of hearing has been put forward for the thicker secondary membrane. Currently it is therefore suggested that the upper membrane is simply an evolutionary remnant of the wing-hinge structure from which the tympanum evolved (Treat \& Roeder, 1959; Yack, 2004).

Finally, as is often the case in biology, one moth ear was examined which exhibits a very wide frequency range, in contrast to that expected. The ear of the greater wax moth (Galleria mellonella) is sensitive to sound frequencies from $20 \mathrm{kHz}$ up to $300 \mathrm{kHz}$ (Moir et al., 2013). This moth listens both for bat echolocation calls and its own courtship calls. However, the upper limit of the frequency bandwidth displayed is far greater than the frequency content of either of these. Therefore it is suggested by Moir et al. that this frequency sensitivity is due to the need for greater mechanical temporal acuity, such that the ability to separate sounds occurring at almost the same time. The response time of a mechanically resonant system such as the moth's ear to a force is inversely proportional to its bandwidth. This means that the moth's large frequency bandwidth provides it with a much 
faster temporal response than a sharply tuned tympanum. The temporal acuity of the greater wax moth was estimated as about $10 \mu \mathrm{s}$, at least half that of the lesser wax moth (Achroia grisella), and six times shorter than some noctuids. This could then aid the moth in carrying out both predator avoidance and possibly courtship. The greater wax moth's ear cannot distinguish between the different frequencies of predator and potential mate. Therefore it must use the difference in time signatures between the sounds. This has been demonstrated in the lesser wax moth, which can determine the difference between the ultrasonic clicks of its courtship calls and hunting bat calls, based on the time period between pulses in each different call. Therefore, it could be that the greater wax moth has such large bandwidth sensitivity in order to maximize this type of capability.

\subsubsection{Locust}

The ears of different species of locust are possibly one of the most studied insect hearing systems, from the work of Michelsen in the 1970s (Michelsen, 1971), to that of many others since (e.g. Gordon \& Windmill, 2015). This hearing system is of great interest because as a passive mechanical system it combines both sound reception and frequency analysis. It is also an insect that is commonly available to any researcher as it is a ubiquitous 'fresh' food for pet reptiles. The work discussed here is primarily based on research into two species, Schistocerca gregaria, and Locusta migratoria.

Locusts can hear frequencies from 100's of $\mathrm{Hz}$ up to over $30 \mathrm{kHz}$. Its ears are found on either side of the abdomen. Its tympanal membrane has several salient features. The largest area is very thin, to less than 1 micrometer, and so transparent. The second smaller area of membrane is much thicker (10's micrometers). Inside the ear sits Müller's organ, which contains the ear's sensory neurons $(\sim 60-70)$, as shown in Figure 6.4. The organ 
connects to the membrane through three cuticular attachments that are clearly seen on the external side of the membrane.

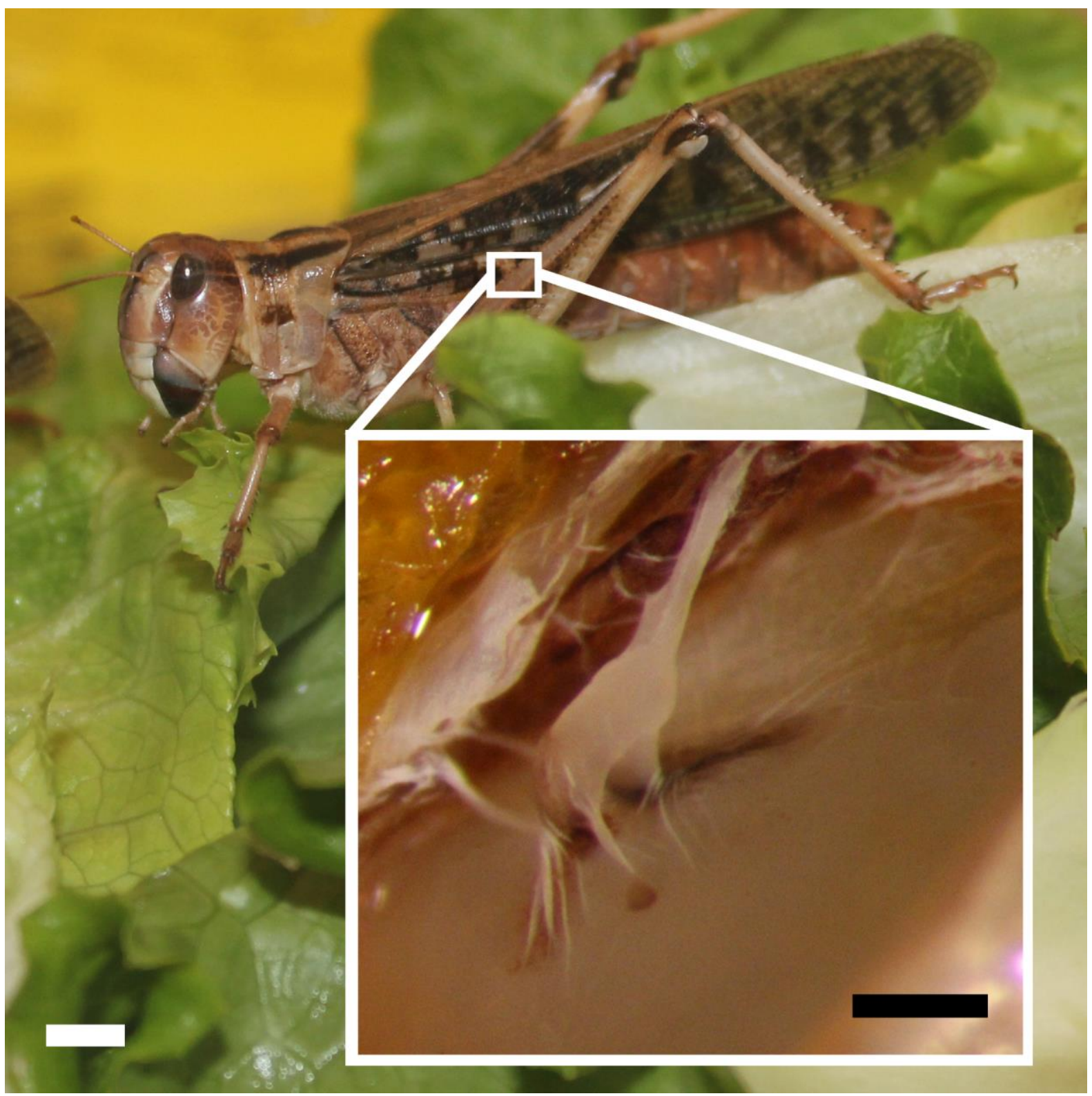

Figure 6.4. The locust has a tympanal ear on either side of the first segment of the abdomen (white scale bar $5 \mathrm{~mm}$ ). Inside the ear sits Müller's organ (inset, black scale bar $150 \mu \mathrm{m}$ ), which contains the ear's sensory neurons. (C) Shira Gordon. 
Locusts are capable of distinguishing different bands of sound frequencies within an overall hearing range. This is achieved through purely passive mechanical means. When sound is incident on the locust tympanal membrane is does not resonate with the mode shapes of a membrane as would be expected. Rather, the sound energy causes the membrane to deflect such that traveling waves are generated running across the membrane (Windmill et al., 2005). These traveling waves are frequency-specific, such that the wave direction perceptibly alters depending on frequency. The frequency also appears to determine the eventual place on the membrane at which the traveling wave disappears. As such, the locust tympanal membrane is an example of tonotopy, whereby the frequency of the sound determines the deflections of the tympanal membrane spatially. This is analogous to the mechanism of frequency discrimination found in the mammalian ear, where frequency specific traveling waves (von Békésy's traveling waves) occur at different positions on the basilar membrane in the cochlea to stimulate different sensory hair cells (von Békésy, 1960). The locust ear only distinguishes a few frequency bands, but it does so without the need for the outer and middle ear structures in the mammalian ear. More recently, the katydid Copiphora gorgonensis has been found to have an ear that is an even closer analog to that of the mammals, and is discussed in the following section (Montealegre-Z et al., 2012).

The traveling wave found on the locust tympanum initiates on the thinner membrane. It then travels across towards the thicker membrane and the connections to Mueller's organ and the sensory neurons. The traveling wave always travels in the same direction, no matter what direction the sound is incident to the locust. The traveling waves caused by high frequency sounds do not propagate onto the thicker membrane, rather they expire at a single point, which is the location of the pyriform vesicle (Fig. 6.4). Lower frequencies propagate into the thicker membrane, such that below $1 \mathrm{kHz}$ the whole membrane is moving, and the 
wavelength is such that it is easier to imagine the membrane moving with a standard mode shape.

In the mammalian basilar membrane the existence of traveling waves has been described as depending on three criteria (Robles \& Ruggero, 2001). Firstly, that the displacement of the membrane exhibit a phase lag in the direction of the wave travel.

Therefore, at a given location the motion of the membrane increasingly lags the motion of its point of origin. In the locust tympanum, this increasing delay with position is found. In the locust it is also a function of frequency as the membrane translates frequency into space. In the frequency domain, the delay is seen as a phase accumulation at high frequencies, which exceeds the high frequency phase-lag expected for a simple resonator. Secondly, the displacement magnitude of the membrane should have an asymmetric envelope around the point of interest, where the wave is seen to compress. This is seen in the locust tympanal membrane, particularly at higher frequencies, where the leading slope of the envelope on the membrane is steeper than the trailing slope. Thirdly, the traveling wave results from the mechanical characteristics of the membrane and, in that sense, is passive. In the locust the traveling wave occurs in freshly dead locusts. Therefore, the motion of the locust tympanal membrane can be described as a traveling wave.

Measurements of the frequency responses of the sensory neurons in Mueller's organ have shown that they correlate with the traveling wave frequencies, so that for example neurons running to the pyriform vesicle respond to frequencies of approximately $9 \mathrm{kHz}$ and above. Other groups of neurons in Mueller's organ are tuned to lower frequency bandwidths, and spatially correlate with the motion of traveling waves at the same frequencies. The path of transduction of force from the traveling wave in the membrane to cause the neurons to generate electrical signals has not been adequately investigated. For example, the pyriform vesicle is clearly deflected by low frequency sounds, as the traveling waves associated with 
any low frequency sound pass through it as they cross the tympanal membrane. It is probably reasonable conjecture to consider that the mechanical behavior of the structures of the pyriform vesicle and about the neurons associated with it relates directly to the actual response of the neurons. Or are the neurons somehow intrinsically tuned to certain frequencies? The only research to attempt to measure the motion of Mueller's organ with respect to sound required sound levels far greater than the insect would usually hear, with a severely dissected preparation (Stephen \& Bennet-Clark, 1982).

It must be noted that no two individual locusts are identical, and therefore, although all show the same characteristic traveling waves, every individual has a slightly different mechanical response. Taking this further, the desert locust $S$. gregaria shows an extreme phenotypic plasticity, exhibited as a trans-generational accumulation of phenotypic changes driven by changes in population density. There are two extreme phenotypes, the solitarious and gregarious phases, which differ extensively in behavior, physiology and also morphology. It has now been shown that solitarious and gregarious locusts have clear differences in their hearing, both in their tympanal and neuronal responses. The shape of the tympanal membrane is different between locust phases, with the solitarious phase having a wider membrane (on one axis of measurement). This correlated with greater displacement of the solitarious animal's tympanal membrane (when given the same sound stimulus amplitude as gregarious phase locusts). This fits with the mechanical expectations for the system, as the larger membrane has greater force applied for the same sound level, as discussed for the moth. The correlation to neuronal response was far less clear. However, this leads back to the questions of how the traveling wave on the membrane interacts with the sensory neurons in Muller's organ (Gordon et al., 2014).

Finally, understanding the mechanisms through which the locust tympanal membrane creates traveling waves is of great interest. In the quest to understand the function of the 
mammalian inner ear numerous mathematical models have been generated. Resources to conduct similar work on the locust hearing system, which many might consider simpler, are rather more limited. However, progress has been made in this area. The typical engineering procedure to model and simulate a system such as a tympanal membrane is to use finite element modeling (Reddy, 2005). In practice this involves creating a two or three dimensional computer model of the system in question. This model is 'meshed' whereby each component in the system is split into numerous blocks of smaller dimension (the elements). The computer simulation then applies physical laws to the elements in the model, working out how each element interacts with those surrounding it. The practical problem is that as the model, and resulting simulation, more closely approach the actual physical biological system, the more intensive the meshing.

In the case of the locust, the tympanal membrane is not a simple flat sheet of homogeneous material. It has a specific problem, in that it has a high aspect ratio; it is very thin in one dimension (thickness of micrometers) compared to the others (diameter in millimeters). This means that a very large number of elements are required for a realistic model based on the actual dimensions of the tympanal membrane. Complicating matters further are all the other related structures, including Mueller's organ, the air sac(s) behind the membrane, the insect's body around the membrane, liquid filled chambers within sections of the membrane (as shown in Malkin et al., 2014), and finally of course the air through which sound travels to the ear.

There are several answers to this problem. First, the power of the computing facility could be increased. However, it is very easy to approach the need for supercomputer facilities even with relatively simple insect ear models. The second approach is to move away from finite element modeling, employing more theoretical mathematical modeling of the concepts, however for the practical biologist (and engineer) this comes at the risk of very quickly losing 
the inherent physical understanding of what is being analyzed. Therefore, the third option is probably the most reasonable, making as many simplifications of the physical model of the locust tympanum as possible in order to be able to undertake an analysis of the system using finite element modeling. And in so doing, hoping to walk the fine line between losing analytical rigor due to over-simplification, and creating a computer model that requires far more resources and time than is feasible to run.

This latter option is the one that has been put to good effect for the locust ear (Malkin et al., 2014). This has compared experimental data with relatively simplified finite element models to show that the locust tympanal membrane is under tension. The combination of this tension, and the change in thickness across the membrane, acts to generate the traveling waves seen moving across the membrane. It also makes it clear that the traveling waves, and the resulting tonotopy, are a purely mechanical effect based on the morphology and material properties of the ear. However, it is noted by Malkin et al. (2014) that their modelling relies on assuming the tympanal membrane is functioning in an isotropic linear-elastic regime although they could certainly be anisotropic and viscoelastic. Thus, the work thus far is probably only a first approximation. This avenue of research can only grow further in the future as the ratio of price to power of computer processing continues to improve.

\subsubsection{Cicada}

Cicadas are one of the loudest groups of animals in the world. They use sound as part of the mating process, ensuring species recognition and sexual selection (Fonseca et al., 2000). Although only the male can produce sound, both sexes have hearing systems. The cicada ear combines a tympanal membrane with a sensory organ containing sensory neurons. The ear contains a surprisingly large number of sensory neurons, varying from 600 to over 2000 in different species. However, the calling song of cicada tends to be centered on a single 
frequency that is amplitude modulated, thus typically producing continuous trains of pulses of sound. The cicada ear differs from that of the moth and locust since the sensory neuron structures do not attach directly to the tympanal membrane. In cicadas, the thin tympanal membrane exhibits a structure called the tympanal ridge, a dark area seen on the membrane, as shown in Figure 6.5. This ridge extends under the membrane through a structure known as the tympanal apodeme. This cuticular extension acts as the mechanical bridge from the membrane to inside the sensory capsule of the organ where the mechanosensory neurons attach to it.

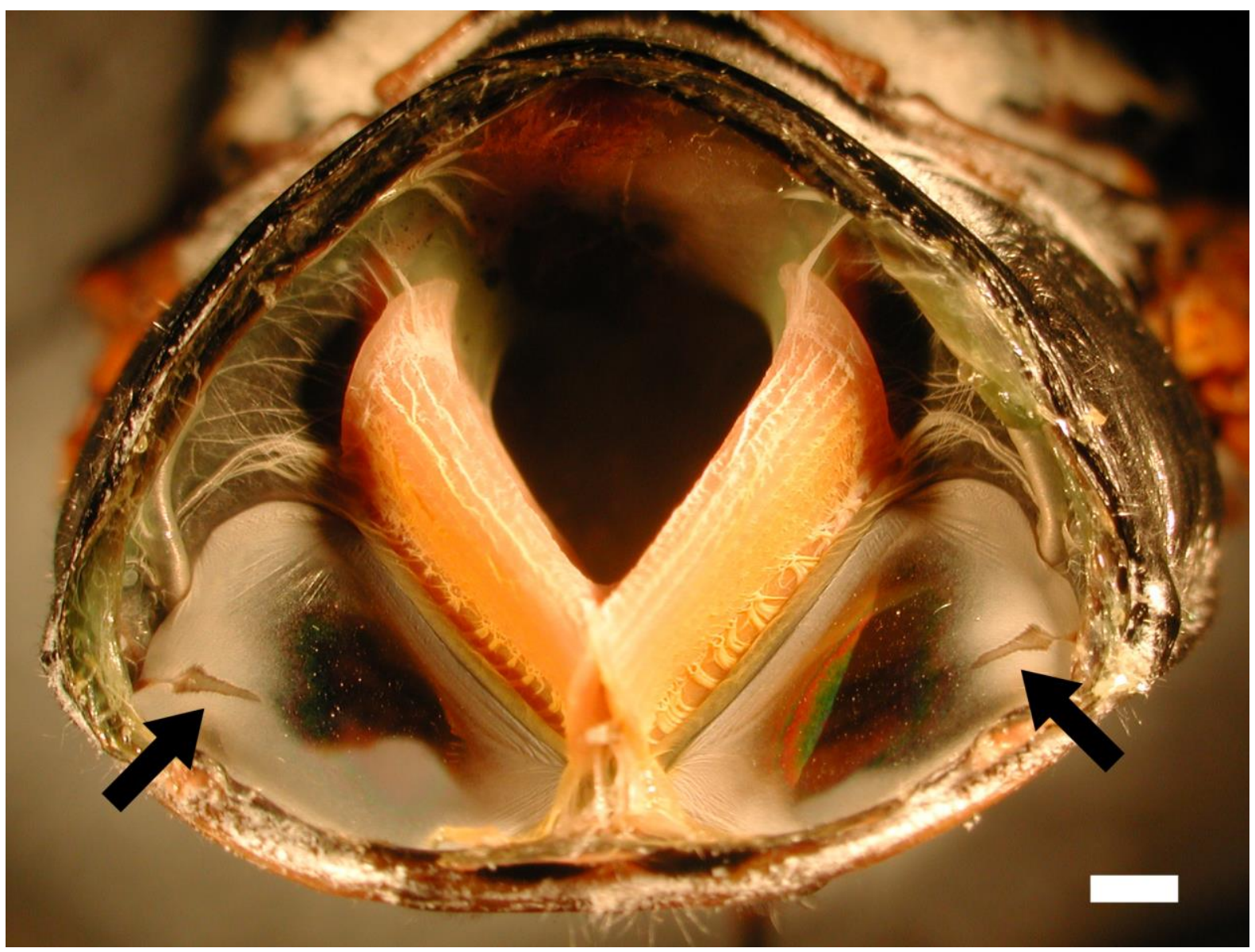

Figure 6.5. The cicada tympanal membrane. This cut-away cross section image shows the two tympanal membranes of a Lyristes plebejus (male), from an anterior view (with the pair of tymbal muscles seen as the $\mathrm{v}$ shaped structure in the center). The ridge structure is clearly 
visible at the edge of each tympanal membrane (located by black arrows). Scale bar $0.5 \mathrm{~mm}$. () Jerome Sueur.

Different cicadas display variations in the mechanical response of their tympanal ear. For example, they may have a traveling wave present on the tympanal membrane and ridge, similar to that seen in the locust, or the membrane and ridge may move with a simple drumlike motion. Sexual dimorphism has also been seen in cicada ears, whereby the male and female ears are mechanically tuned to different frequencies ranges, presumably relating to selective pressures acting in different directions, linked to the different roles of each sex in sound reception and production (Sueur et al., 2010). However, in all cases the cicada's tympanal membrane and ridge act to mechanically focus the incident sound energy to drive the activation of the sensory neurons.

Force is applied to the mechanosensory neurons through the motion of the tympanal apodeme. The apodeme appears to be a relatively thick cuticular structure, and appears to act as a lever, with the external membrane and ridge at one end, and the sensur y munvis a un other. The apodeme appears to be stiff, and does not bend, at least along the portion of the structure which has neuron connections. However, the mechanical frequency response of the apodeme is tuned about the main frequency of the cicada's calling song (Windmill et al., 2009). It thus appears to act as a passive frequency filter within the cicada ear, meaning that only energy related to the calling song reaches the sensory neurons. Also, the amplitude of the motion of the apodeme is significantly less than that of the external membrane. Thus it is also possible that one purpose of the apodeme is to reduce the amount by which the sensory neurons are stretched. It is not clear if this means, as in a simple lever system, the force applied to stretch the neurons is increased in relation to the reduction in the amplitude of motion. As such this could be some form of impedance matching, translating the motion of 
the thin and light tympanal membrane (which seeks to match the impedance of air), to the stretching motion of the large number of sensory cells. It should be noted, however, that experimental data on the mechanics of the cicada apodeme are so far available for only a single species (Windmill et al., 2009).

Finally, it is also not clear why the cicada has so many (hundreds) of mechanosensory neurons, especially given that the cicada calling song is an amplitude modulation of a single frequency. Electrophysiological measurements have been undertaken which show that interneurons following the sensory neurons in a cicada hearing system carry out a frequency discrimination (Fonseca et al., 2000). However, no equivalent experiments have been carried out on the sensory neurons to examine whether they are frequency tuned in some manner. As discussed in Section 3 certain insect ears are "active," such that they are motile, and can add energy into the system to make ear structures move more. So, the question is open. Could the large number of neurons be related to frequency tuning? Or maybe, if motile, to add mechanical energy into the system? Or some other reason as yet to be discovered?

\subsubsection{Katydid}

Katydids (or bushcrickets) are another insect that uses sound as part of the mating process, as males sing to attract conspecific females (Montealegre- $\mathrm{Z}$ et al., 2015). The sound frequencies exploited by different species of katydids vary across a huge range from 2 to 150 kHz. Further, in addition to conspecific signals, katydids are exposed to many other sounds, including ultrasound produced by bats to hunt and navigate at night, as well as the sounds produced by other nocturnal mammals that eat insects (i.e. many tropical species of katydid are nocturnal). Thus, the katydid ear has evolved within the context of communication and predator detection, meaning that many species can detect a wide range of frequencies. 
The katydid ear is found in the leg of the insect, with one in each foreleg tibia, as shown in Figure 6.6. Each ear actually has a pair of tympanal membranes, one anterior and one posterior. Each tympanal membrane also has a thicker section, known as the tympanal plate. Behind the membranes is a trachea, filled with air, which divides into two branches at the ear, one for each tympanal membrane. The ear is asymmetrical as the anterior branch of the trachea takes up a large part of the dorsal ear surface. In some species the trachea plays an important role in the transmission of sound to the ear, as sound enters through a spiracle. Depending on species, there are various suggestions of different adaptations of the tracheal system, in concert with the pair of tympanal membranes relating to the reception, transmission and amplification of sounds. There is also great variation in the external morphology of the ear around the tympanal membranes, whereby in different species the tympanal membranes are either completely exposed, or partially covered by cuticular structure, or only one is exposed. It has been suggested that the covering structures function as sound guides to enhance directional hearing in those species. Back within the ear, the mechanosensory neurons sit in a long structure called the crista acustica. This structures sits on the dorsal wall of the anterior tracheal branch. It is surrounded by a fluid filled cavity, described as the auditory vesicle. The sensory neurons in the crista acustica are then tonotopically organized. However, one of the most interesting morphological and mechanical points regarding the katydid is the fact that the sensory cells are not in direct contact with the tympanal membranes. 


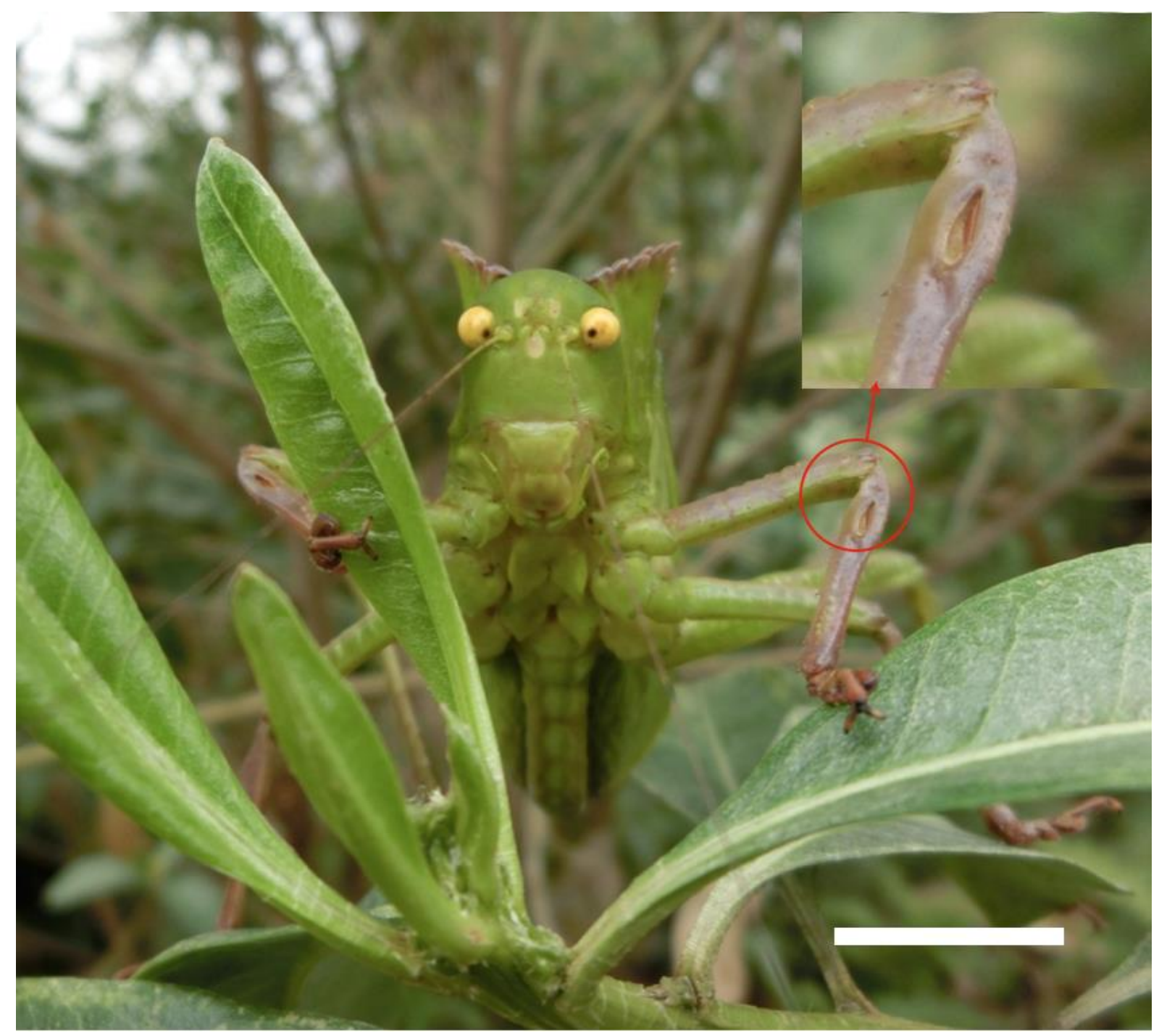

Figure 6.6. The giant katydid Steirodon careovirgulatum, with the ear, found on the foreleg tibia (inset). Scale bar 30mm. (C) Fernando Montealegre-Z.

As noted previously in this section, the sensory neurons sit within a fluid filled cavity, and are not in direct contact with the tympanal membranes. Therefore, in the ear of the katydid a mechanical transduction process is required to convert the tympanal membrane vibrations due to sound into mechanical energy in the fluid in order to drive the sensory cells. As discussed in Section 6.1, the impedance of air, cuticle and fluid is different, therefore the katydid ear must undertake a process of impedance conversion, in a similar manner to that found in the mammalian ear. The answer is a lever system, whereby the motion of the 
tympanal membranes acts to drive the fluid, and then the fluid acts to drive the sensory neurons. This is important because the tympanal membranes act as simple resonant membrane structures, i.e. with a central resonant frequency, whereas the crista acustica is a tonotopic system, with different sensory neurons along the crista acustica activated by different frequencies. In fact the crista acustica displays the traveling wave motion found in the mammalian basilar membrane, and the locust tympanal membrane.

Unfortunately, this is complicated somewhat by the fact that different species of katydid appear to have variations on this basic concept (Montealegre-Z et al., 2012; Palghat Udayashankar et al., 2012). In some katydids the tympanal membrane and plate move in phase. In this case it is suggested that pressure waves traveling in the trachea activate vibrations of the crista acustica internally. So as the tympanal membranes move with sound, the change in pressure within the trachea causes the crista acustica to move. The second case is where the tympanal membrane and plate are seen to move out of phase. In this case it is put forward that the tympanal plate is acting as a lever, like the ossicles in the mammalian middle ear. So here the change in air pressure causes the tympanal membranes to move, and the mechanical connection between a membrane and associated plate then acts as a lever, such that the motion of the membrane is mirrored in the plate. The plate sits in contact with the fluid filled cavity within which the crista acustica is found. So the plate then couples its motion to the fluid filled cavity, causing pressure waves within the fluid. It is then these pressure waves in the fluid that cause the sensory neurons in the crista acustica to be driven to move. In this second case it is clear that the system is then a very close analogy to the mammalian ear. The air driven tympanal membrane (eardrum) mechanically couples to a stiff lever system (the middle ear), which then couples to a fluid system, wherein the motion of the fluid causes the activation of the sensory neurons (the inner ear). 
As mentioned previously, the sensory neurons within the crista acustica are tonotopically arranged, such that the sensory organ can discriminate frequencies across a range (dependent on frequency). This is seen through the formation of traveling waves through the crista acustica, with different frequencies relating to traveling waves being produced at different positions along the structure. Again this appears slightly more complex across species, relating directly to the differences between how the crista acustica appears to be driven. In the first case, where the crista acustica is driven by the change in sound pressure in the trachea, it appears that the traveling waves form whether the fluid in the cavity behind the crista acustica is present or not. In the second case, where the tympanal plate motion causes pressure waves in the fluid, it appears that removal of the fluid stops the formation of traveling waves in crista acustica, as expected. It is suggested that the traveling waves are a result of the morphology and mechanical properties of the crista acustica. This seems appropriate as the crista acustica is wide at one end, narrowing along its length. As noted before, this is again a close analog to the mammalian ear, where the basilar membrane not only fulfils the same function, and displays the same vibrational motion, but also has the same type of change in morphology.

Finally, it also appears that the katydid has a form of mammalian 'round window', whereby the pressure change input to the fluid system is output, as a form of pressure release, as the fluid is incompressible. In some katydid the proximal end of the fluid filled cavity has a narrow connection with the hemolymph channel, such that the pressure input relating to the sound is released into the insect's hemolymph (Montealegre-Z \& Robert, 2015).

\subsubsection{Sound Direction}

Directional hearing is usually accomplished through a mechanism that compares the sound input between two ears, relying on either interaural time or intensity differences, ITD 
and IID respectively. To accomplish a timing difference measurement, the distance sound must travel to reach each ear independently must be large enough for a detectable time delay to exist between the ears. For a small animal like an insect, the distance between ears is generally smaller than the sound of interest's wavelength such that a time delay detection approach is not feasible.

Despite this, when the distance sound travels to reach the ears fall within half a wavelength of the incident sound, animals may be able to make a phase comparison. In this circumstance, the sound pressure level is relatively similar at either ear, and so animals could plausibly register phase differences of the wave as it passes around the body. In addition, the air chambers in an insect's body form an internal acoustic connection between the tympanal membranes at each ear. Therefore, whilst a force is exerted externally on a tympanal membrane due to the external sound pressure changes, a secondary, attenuated, sound pressure change contribution from the incident sound is applied from the inside of the membrane via the air chamber pathway (as in a pressure-gradient receiver).

The wavelength of a sound wave in air decreases as the frequency increases. Therefore as frequency increases, there is a critical frequency beyond which the insect is unable to decipher the phase difference between the ears relating to the incident sound wave. For frequencies greater than this value, a difference in sound pressure level must instead be detected. Theoretically, in this second case the distance between the ears must be greater than half the wavelength of the incident sound, with this half wavelength value corresponding approximately to the critical frequency. With higher frequencies, the insect's ears are functioning solely as pressure-difference receivers, whereby the internal sound pressure is unaffected by the change in external pressure. Increasing frequencies will result in smaller wavelengths and therefore larger sound level differences between the ears as the insect body acts as a barrier to sound propagation. 
At lower frequencies, where the distance between the insect's ears fits within half a wavelength, the direction of the sound is determined by the phase of the sound wave arriving at both the external and internal (from the contralateral ear) sides of the membrane (pressuregradient receiver); the animal's body is too small to significantly affect the sound wave as it passes and all or most of the sound diffracts easily around the body. However, for higher frequencies, sound will not be able to diffract around the animal's body and so a partial acoustic shadow is created, with a diminution of sound pressure level on the contralateral side to the incident sound. In addition, the body creates reflections that change the sound field. When sound originates from directly in front of the animal no difference in phase or sound level will be detected due to the bilaterally symmetric placement of the ears. To complicate matters, sound arriving from one of the sides will create interference both from the length of the body and from the width around the body.

The accumulation of the constructive and destructive interference patterns is commonly measured by comparing the sound level within a free sound field to the altered sound level when there is an obstruction (aka body). Therefore, directional hearing is often discussed as the changes in sound level both between the ear locations and as if there was no body obstruction.

\subsubsection{Ormia}

The parasitoid fly Ormia ochracea is a parasite of crickets at the larval stage. As a consequence gravid females deposit their larvae on a host cricket which located through phonotaxis to the male cricket's mating call (Miles et al., 1995). This parasitic lifecycle imposes a significant evolutionary constraint on the flies, as their body size must be limited to being less than that of their hosts. This means that the auditory organs of $O$. ochracea are only separated by a short distance, such that there is less than $0.5 \mathrm{~mm}$ between the fly's 
tympanal membranes, which in turn are very small. The cricket's mating call has a relatively pure frequency tone between $4.5 \mathrm{kHz}$ and $5.2 \mathrm{kHz}$ (so wavelength of approximately $70 \mathrm{~mm}$ ). This presents significant challenges for sound source localization as the ITD is $1.45 \mu$ s at maximum azimuth of $90^{\circ}$ and the IID in the sound pressure is extremely low. Nevertheless, this tiny ITD the parasitoid fly has been shown to be able to localize cricket song to an azimuthal accuracy of $2^{\circ}$. This is comparable to the directional hearing of humans who are able to rely on much larger ITD and IID given the distance between their ears. Extensive studies of the $O$. ochracea auditory system have shown that the tiny ITD cues are amplified by means of a flexible mechanical coupling between the two sensory organs.

The ears of a parasitoid fly are unusual in that both the tympanal organs are contained within a single, undivided air-filled chamber. The auditory system is located on the front face of the thorax with a pair of cuticular membranes serving as the tympana. The two tympanal membranes are connected through a cuticular structure referred to as the intertympanal bridge. The intertympanal bridge terminates near the center of each tympanum in a depression (the tympanal pit) that is also the attachment point of the auditory receptor neurons. It is this cuticular bridge that provides the mechanical basis for the directional sensitivity. Measurements of the tympanum using laser vibrometry have shown that the membranes vibrate with amplitude differences of approximately $12 \mathrm{~dB}$ and time differences in the order of $50 \mu \mathrm{s}$ at maximum azimuth (at $6 \mathrm{kHz}$ ).

The coupling of the ears can be shown by manually applying a force to one membrane, resulting in the intertympanal bridge rocking about the point halfway between the pits. When stimulated acoustically both sides of the bridge are driven by two forces of equal amplitude but slightly different phase. The overall motion of the bridge can then be decomposed into two natural modes of vibration. The first is a pure rocking mode about the center point of the bridge, while the second is a translational mode with both ends of the 
bridge moving in-phase while the intertympanal bridge bends in the middle. The response of the hearing system at any frequency can then be described by a linear combination of these modes.

The ability of the incident sound pressures to drive each of the modes depends on the relative phase of the pressures acting on the tympana. The instantaneous sound pressure at each ear is equivalent to two sinusoidally varying point forces which are very slightly out of phase (see Fig. 6.7). The difference in these two forces provokes the rocking motion of the bridge while the sum of the forces provokes the translating mode. The instantaneous amplitude difference between the two forces then clearly depends on the wavelength of the incident sound and the natural resonance frequencies of each of the modes. In O. ochracea at $2 \mathrm{kHz}$ the phase delay is approximately $1^{\circ}$ resulting in a very low IID and a dominant translational mode. At $5 \mathrm{kHz}$, the frequency of the cricket mating call, the phase delay is larger $\left(\sim 2.6^{\circ}\right)$ which provokes a larger rocking mode supported by the proximity of this frequency range to the natural resonance of that mode at $7 \mathrm{kHz}$. In higher frequencies the phase difference is greater, but the rocking mode is depressed by being driven above the natural frequency, while the translational resonance is approached resulting in both modes being equally strong in the system. Therefore, O. ochracea is critically tuned to have excellent discrimination of directional sound cues at the frequency of the cricket mating call. And so, through the addition of a mechanical link between the two tympanal membranes the O. ochracea is capable of extremely fine directional hearing. 

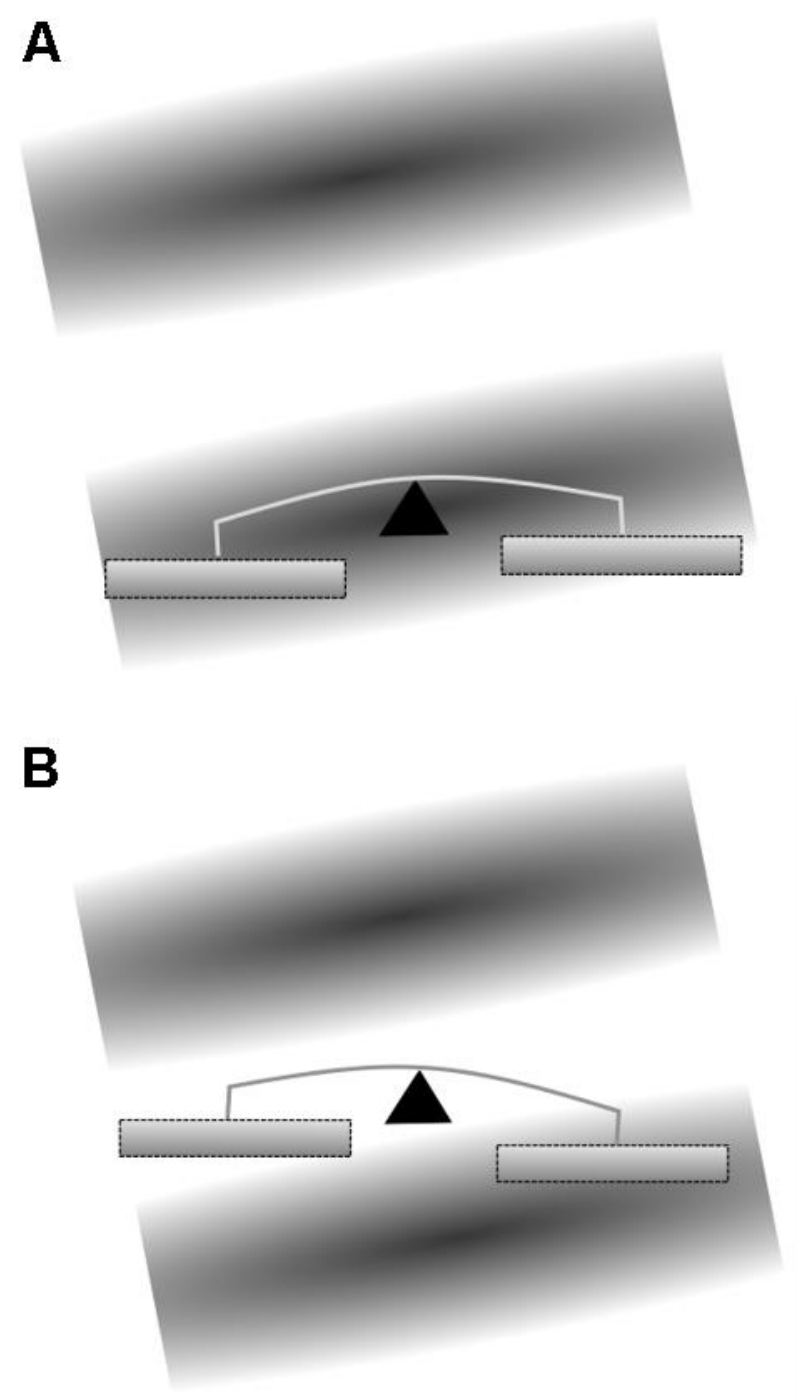

Figure 6.7. The Ormia ear mechanism. The ear's two membranes are connected by an intertympanal bridge. The bridge motion has two natural modes of vibration: 1) A rocking mode about the bridge center point (triangle); 2) A translational mode with both membranes moving in-phase while the bridge bends at the center. A sound wave, depicted by shading (see Fig. 6.1) causes the system to move, with the difference in pressure between the membranes stimulating the rocking mode and the sum of the pressure on the membranes stimulating the translational mode. A. At one quarter cycle the force from the total pressure is at a maximum while there is no pressure gradient between the membranes, reinforcing the movement of the ipsilateral membrane while repressing the movement of the contralateral 
membrane. B. The forces from the pressure gradient and total pressure on the membrane are 90 degrees out of phase. At three-quarters cycle the pressure gradient is at a maximum while the total pressure is reduced to zero by the angle of the membrane to the sound wave origin. (C) Andrew Reid.

\subsubsection{Other Insect Ears}

Hearing in insects has evolved multiple times (Yack, 2004). Whilst the passive mechanical response of a number of insect ears has been discussed in the preceding sections, and active responses in other insects will be examined in the next section, this is by no way an exhaustive analysis of the passive mechanics of insect hearing. Current understanding depends on the resources that have been invested in the research required. Hearing in various other insects has been examined to a greater or lesser degree. A number of other insect hearing systems have been studied, two examples of which, the mantis and the weta, highlight the diversity of mechanical adaptations in insect auditory systems.

The mantis hearing system is unique, as it contains only a single ear, and is often referred to as the cyclopean hearing system (Yager \& Hoy, 1986). The vast majority of mantids can hear only ultrasound frequencies (typically $30-50 \mathrm{kHz}$, but sometimes extending towards $100 \mathrm{kHz}$ ). The main function of the ear therefore appears to be the detection, and so avoidance, of predatory bats. This single ear of the mantis has two tympanal membranes located in a deep cuticular chamber that is found in the ventral midline of the metathorax. The teardrop shaped tympanal membranes face each other from the walls of the groove. The mechanics of the membranes are interesting as they also utilize a traveling wave. It is also most likely that the chamber performs an acoustic function. However, research into the ear of the mantid is ongoing, and is sure to reveal more about this unique ear. 
As a member of the Ensiferan group, the weta is related to the katydids and crickets, and so also has an ear on each foreleg tibia (Field et al., 1980). Weta produce sound by stridulation, and their hearing is typically tuned over a relatively narrow frequency range relevant to acoustic intraspecific communication. As in the other Ensiferans the ear is composed of two tympanal membranes, one anterior and one posterior. The tympanal membranes have two distinct regions, a darkly shaded thick inner region surrounded by a thin transparent region. The inner region oscillates as a stiff plate driven by the surrounding region. As the complete tympanal membrane vibrates in a simple drum-like mode this only provides an initial frequency discrimination, based on the membrane's drum-like response. The weta ear also has an adapted trachea, providing an air cavity behind the tympanal membranes, and a crista acustica containing the sensory neurons.

The unique part of the weta ear is that the fluid in the channel that the crista acustica is sited in comprises a previously unknown form of lipid, rather than the hemolymph found in katydids (Lomas et al., 2012). Furthermore, the lipid is synthesized in situ by a structure known as the olivarius, distinct from the fat body. Removal of the lipid reduces the neural auditory sensitivity of the ear. Thus the lipid channel is thought to act as a relatively solid mass preventing the crista acustica and trachea from moving dorsally. Instead, the trachea is thought to be constrained to expand laterally, stretching out the ends of the crista acustica and thus activating the sensory neurons. Clearly, questions still remain regarding this system, for example whether the lipid's function is purely a passive mechanical one. Therefore research into this system continues.

\subsection{The Active Mechanics of Insect Ears}

While properties of insect ears that rely on structure - or form - to determine their acoustic function have been discussed previously, there is another mechanism by which 
acoustic properties can be changed that doesn't rely, intrinsically, on ear structure. This mechanism, known as active hearing, uses metabolically dependent processes to add energy to an existing acoustic oscillator. Additional vibrational energy derived endogenously has the effect of conditioning the acoustic response of the ear for whatever purpose, typically amplification of weak signals, and increasing sensitivity.

There is a wealth of studies in the scientific literature on active hearing in the animal kingdom, the content of which cannot be reproduced in its entirety here - the reader is advised to read Manley et al. (2008) and Nadrowski et al. (2010) for more detail. Instead, the general principles of how active hearing can improve audition at the periphery from a physical perspective will be described, followed by examples of active hearing in the insects which act as model systems for their auditory behavior.

6.3.1 A Reduced Physical Description of the Addition of Active Properties to an Ear

A basic 'ear' can be considered. Ears in nature are first and foremost passive oscillators, driven by fluctuations in fluid pressure or particle velocity in the medium through which sound propagates. As oscillators, they can be approximated as driven damped harmonic oscillators - an elementary physical model of an oscillator in which dynamic behavior is determined by three parameters (reducible to the first two) - stiffness $k$ of a linear restoring spring, linear viscous damping $b$ of the medium in which the oscillator moves, and the mass $m$ of the oscillator.

Oscillators of this type are linear, which in this context means that they will oscillate at the same frequency of the impinging sound field, and their response is proportional to the amplitude of the sound field. They also exhibit resonance - a preferred frequency that 
induces strong vibration compared with other frequencies. For insects, linear passive ears means that the ear should a) vibrate with no distortion in response to a sound, or put another way, the ear can only vibrate at frequencies that were present in the impinging sound, b) exhibit some resonance, whether pronounced or damped, which could be used for selectivity and c) respond the same way to a sound, independent of any previous sounds that may have impinged upon it. As such, this basic insect ear, when driven with a pure tone, should vibrate sinusoidally at a predictable consistent amplitude.

It will not be possible to describe the many fine details of the responses of passive oscillators to sound. Instead the focus is to consider this basic ear already vibrating sinusoidally at a fixed amplitude as a result of a driving sinusoidal force from sound. A graph of the instantaneous displacement of this vibration oscillation versus the instantaneous velocity traces out a circle (see Fig. 6.8). In time the oscillator rotates around this circle - one cycle of this circle is one full cycle of oscillation. It is possible to imagine an active process that somehow allows the addition of energy at any point in the cycle; for example, as the oscillator traces this circular path, there is some way of 'pushing' it around. Depending on how this is achieved, adding energy to different parts of the cycle has the equivalent effect of changing the effective parameters of the oscillator - the stiffness and the damping components. For example, at the point of maximum velocity, the displacement is zero. If an impulsive force in the direction of motion contributed energy at this point, then it would oppose the viscous damping of the medium on the oscillator. Consequently, it would seem that the viscous damping was effectively reduced. A similar argument can be made for when the oscillator velocity is zero and the displacement is maximum - energy added at this point would affect the apparent stiffness. 

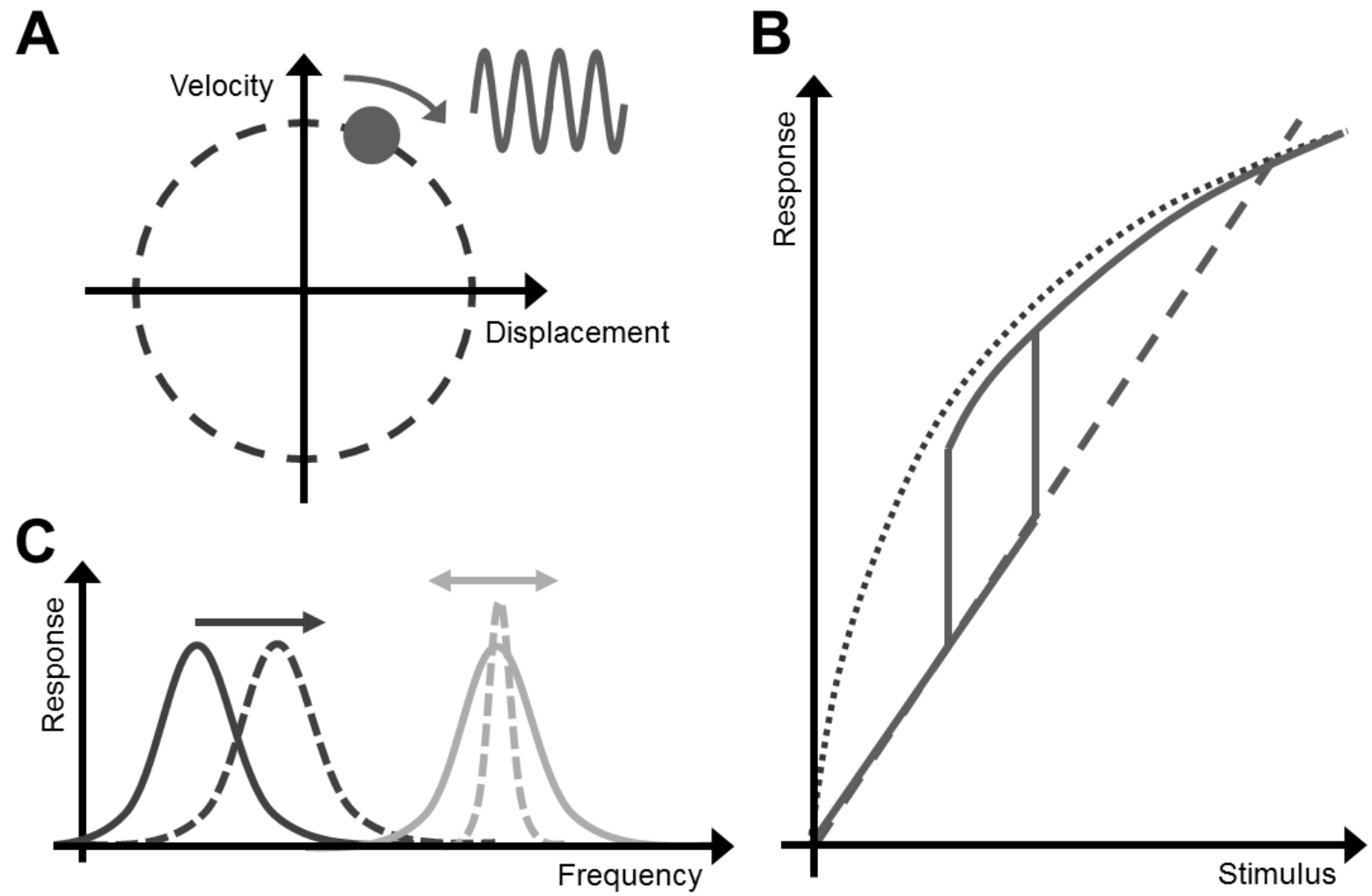

Figure 6.8. Active insect ears. A. Phase-space plot of harmonic motion. In time a sinusoidal oscillation traces out a circle. B. Types of active nonlinear responses - Grey dotted line is a typical vertebrate hair bundle response, or that of drosophila or the tree cricket. Solid line is the mosquito nonlinearity, indicating the hysteretic response. The dashed grey line is a linear response. C. Energy added at different points of the cycle can either shift the resonant frequency (dark grey) or change the effective damping and sharpen or desharpen the frequency response (light grey). In practice it is some combination of each. @ Joseph Jackson.

This rather abstract thought experiment demonstrates how active cycle-by-cycle processes can affect the effective parameters of a simple oscillator, influencing stiffness and damping which, in turn, influence the response of the oscillator - in essence the passive oscillator has been altered and so its response to a sound stimulus will be different. 


\subsubsection{Self-oscillation}

In this basic ear, imagining an active process that can push and pull the oscillator allows predictions of how this would change the effective stiffness and effective damping of the ear. If such an active process is too strong, the effective damping becomes zero, or even negative. Under these circumstances, in a basic ear, in the absence of a driving force, the active process could drive autonomous oscillation. This is possible, and is considered the gold standard indicator of active hearing - understandably, as in the absence of sound, there is no external energy input into the ear, and thus it can only oscillate with an active process.

\subsubsection{The Oscillatory Instability}

That an active ear is sometimes capable of autonomous oscillation allows a generic mathematical model to be used for the dynamic behavior of this nonlinear oscillator. Such a general model derives from the normal form for a Hopf bifurcation (Pikovsky \& Kurths, 2003), and was successfully used to describe various phenomena in the dynamics of auditory hair bundles (Camalet et al., 2000; Eguiluz et al., 2000). This model easily explains the power-law behavior of hair bundles exposed to weak acoustic stimuli. Conceptually, the predictive power of this normal form is restricted to generic properties of acoustic nonlinear sensors close to an oscillatory instability. Therefore it cannot provide detail about a particular type of sensor. However, given that such properties of active ears are generic, they can be considered important experimental phenomena that betray the presence of an active mechanism in ears. 


\subsubsection{Active Insect Ears}

In the insects, active hearing has been found notably in mosquitoes, Drosophila, and a tree cricket (Oecanthus henryi). Self-oscillation is a characteristic property of these ears, in common with mammalian and frog saccular hair bundles (Kemp, 1978; van Dijk et al., 1989). There is also evidence to suggest locusts and moths have some nonlinear active aspect to their hearing, evidenced by acoustically evoked distortion in their tympanal membranes. It seems unlikely that active hearing is restricted to these insects only. For example, little study has been made of hearing in the nematocera, the suborder to which mosquitoes belong despite many of this suborder having plumose antenna that could in principle be used for hearing. It is anticipated that new discoveries of active hearing in insects will occur in due course - in the meantime what follows is a description of some well-known active hearing systems in the insects.

\subsubsection{Antennal Ears}

Mosquitos and Drosophila, among other insects, have antenna that act as acoustic oscillators. At the base of these antennae is Johnston's organ, a chordotonal organ whose function has evolved from proprioception to acoustic sensing. This organ contains many mechanosensory units called scolopidia comprising ciliated neurons (e.g. Boo \& Richards, 1975). Cilia within these scolopidia are thought to be capable of generating force, thus providing an origin for energy pumping into the oscillating ear. What follows is a brief description of the two model systems for active antennal hearing in insects. 


\subsection{Mosquito}

The male mosquito ear is a plumose antenna, at the base of which is a pestle-shaped organ called the pedicel in which lies Johnston's organ (Clements, 1999). It can be considered a beam supported by a spring at the base and as such its first mode of vibration is a simple rocking motion of the antenna within the pedicel. Thus, the antenna does not bend. Johnston's organ in the mosquito consists of some 16000 mechanosensory neurons, a very large number and probably the key to the unusual nonlinear behavior of the mosquito ear. This ear can be well approximated as a harmonic oscillator, with damping caused by the plumose antennae moving through air, and stiffness dictated by the joint between flagellum and Johnston's organ. The oscillator has a primary resonance at approximately the same frequency as the female flight tone - the male ear is used to listen for a flying female.

The antenna of the elephant mosquito Toxorhynchites brevipalpis exhibits autonomous oscillation both spontaneously and elicited with microinjection of, for example, Dimethyl sulfoxide (DMSO). Such oscillation is approximately sinusoidal. Experiments show that when stimulated with sound mimicking a female acoustic signal, the antenna behaves nonlinearly above a certain sound level threshold (Jackson \& Robert, 2006). A bistable response exists where the antenna can oscillate with two different amplitudes. Which amplitude is 'chosen' as the stable oscillation depends upon whether the sound stimulus intensity was approached from a louder sound or a quieter sound - a phenomenon that has behavioral implications.

Antennal nonlinearity has been shown to involve significant changes in the effective damping of the antennal oscillator, with a small change in the effective stiffness. Such an effect requires energy input on a cycle-by-cycle basis and the huge number of ciliated mechanosensory neurons is undoubtedly required to fuel this mechanical response. One 
potential indicator of active hearing is therefore the presence of an unusually large number of potentially motile sensors in an insect ear.

\subsection{Drosophila melanogaster}

Drosophila exhibit active hearing too, but with a contrasting result compared to the mosquitoes. Drosophilid antennae are of a different form to the mosquito, but the basic principles are much the same - some external paddle-like oscillator damped by air, and sprung at the base of the antenna to Johnston's organ. In this animal, the chordotonal organ has a few hundred mechanosensory neurons.

The first evidence for active hearing in Drosophila is the ability of the antenna to selfoscillate. However, rather than an almost sinusoidal vibration as seen in the mosquito, the Drosophila antenna exhibits a strong nonlinearity and the resulting self-oscillation is highly non-sinusoidal - it vibrates as if it were switching between two extreme displacements (Göpfert \& Robert, 2003).

The antenna exhibits an elevated mechanical response in vivo compared to a hypoxic state. When stimulated with sound, Drosophila antenna exhibit a strong change in resonant frequency. As such, the effective stiffness of the oscillator is strongly influenced by active processes, in contrast to the mosquito (Göpfert et al., 2005). Again, the suggestion is that energy is added cycle-by-cycle and in this case is predominantly affecting the effective stiffness.

The response to single-frequency stimuli demonstrates power-law responses consistent with the generic models for active ears. As such, the Drosophila ear is an excellent model system to aspects of the evolution of active hearing in finer detail. In particular the 
similarities between models for Drosophila hearing and vertebrate hair cells, and the amenability of experimentation on fruit flies, make the study of Drosophila hearing an exciting avenue for future research (e.g. Todi et al., 2008).

\subsubsection{Tympanal Ears}

Active hearing in insect tympanate ears has also been discovered, and evidence exists for active hearing in orthopterans and lepidopterans (e.g. Coro \& Kössl, 1998; Kössl \& Boyan, 1998; Windmill et al., 2006; Mhatre et al., 2013; Mora et al., 2015). As discussed previously, tympanal ears are drum-like sensors that detect pressure fluctuations, in contrast to antennal ears, which detect fluid flow. Tympanal ears have mechanosensory organs attached directly or indirectly to the tympanal membrane. Again, the interplay between the mechanosensory attachment and the passive tympanal membrane is thought to be responsible for enabling active phenomena.

\subsection{Tree Cricket}

A discovery by Mhatre et al. (2013) showed a very strong active hearing phenotype in the tree cricket Oecanthus henryi. These ears are present on the foreleg of the cricket, and are typically orthopteran. The remarkable aspect of this discovery is how well the ear matches the canonical properties of active hearing: strong inducible self-oscillation that is approximately sinusoidal, compressive nonlinearity with power laws consistent with a Hopf bifurcation model, and distortion that is dependent on physiological condition. Despite its very recent discovery, the tree cricket should be considered a model system for active hearing due to it being expressed strongly in this animal. 


\subsection{Locust}

The locust is a tympanal system that exhibits a vibrational tonotopy phenomenologically equivalent to the traveling wave observed in the mammalian cochlea. Furthermore, there exists evidence that the tympanal ears of locusts are also active. Unfortunately, locust hearing does not exhibit strong phenomena associated with active hearing. There is no evidence of power law responses, and no evidence of self-oscillation. However there is evidence for distortion (Kössl \& Boyan, 1998). At first glance, distortion without self-oscillation or power-law responses would imply a nonlinear ear, but not necessarily an active one. However this distortion is shown to be metabolically dependent. Further research is needed to examine the exact nature of activity in the locust ear and what benefit the animal enjoys from it.

\subsection{Noctuid Moths}

Of course, nothing in nature is straightforward, and nonlinear hearing can be present in an unexpected way. The previous examples describe effects from cycle-by-cycle force feedback into an oscillating sensor allowing changes in the effective damping and stiffness. However, there are alternative ways to change, for example, the stiffness. In the moth Noctua pronuba, the resonant frequency - and so the stiffness - of the tympanum appears to increase in response to bat-type stimulus (Windmill et al., 2006). This phenomenon has since been observed in various moth species (e.g. Mora et al., 2015). This stiffness change is inferred from a clear change in the resonant frequency of the tympanum. However it appears that this stiffness change is not a cycle-by-cycle feedback resulting in an effective change of stiffness - rather it appears that it derives from a direct manipulation of the stiffness of the tympanum. Thus examples of changing stiffness, or indeed damping, are not sufficient to state the 
existence of active hearing. Nor is the presence of a power law, or distortion, which may only indicate nonlinearity. The only true proof of active hearing is either self-oscillation, noisy oscillations, or metabolically dependent nonlinearity that result in amplification or distortion.

\subsection{Conclusions and Future Directions}

The preceding sections of this chapter have hopefully provided an inspiring, if very brief, introduction into the mechanical specializations of insect ears. It is in no way a complete treatise of the subject, there are insect ears not mentioned, or mentioned so briefly that those researchers who work on them will question this treatment of the subject. For example, some might ask what happened regards the hearing system of the cricket? Even for those insects which have been dealt with in some depth, this chapter can only act as an introduction. There is a very large body of research for the aspiring researcher to work their way into.

It is clear that researchers are learning more and more about the complexities of what some would call 'simple' hearing organs. The ears of different insects have evolved in various mechanical ways to carry out useful functions relating to the survival of the species, through communication and defense. These hearing organs are capable of similar feats of sensitivity and processing to those found across the hearing animals. In doing so they can mechanically process sound amplitude, frequency content and direction, as appropriate to their ecology. This has led to many interesting variations across these themes. So, an insect might only need to pick out one frequency, but does it need to know with great accuracy where it comes from (Ormia), or just that the presence of that sound frequency equates to an immediate threat (moth) or a mate (possibly the same moth!)?

Through the years the capability of researchers to utilize technology has helped generate much progress in the area. The use of a laser vibrometer to measure the nanometer 
scale motion of a hearing organ is now almost ubiquitous. This is now being coupled with advances in microscopy, in particular the availability of x-ray micro-tomography $(\mu \mathrm{CT})$, which allows the visualization, and measurement, of hearing organ structures and surrounding body parts in three dimensions. Thus it is now possible to see how the internal structures, air channels and other internal organs sit in a three dimensional space around the ear. The third area that is slowly advancing is the use of computer modeling and simulation to understand the dynamics of the mechanical function of the ear. Unfortunately this is still limited, even with the continuous increases in computer power. At the time of writing it is not yet possible for a 3D microCT file to be loaded into a 3D computer modeling suite, and after some manipulation by a researcher a 3D simulation of the mechanical function of the ear be produced and directly compared with empirical measurements from a laser vibrometer. However, that day is getting closer, and researchers in the future will have access to such tools.

Unfortunately, the panacea, at least for some, described above misses two vital points. The first one is that for any computer modeling and simulation to make sense the material properties of the structures must be known. This is far easier said than done. Currently mechanical models and simulations tend to take a first approximation approach, assuming the system is neatly isotropic and in a linear-elastic regime. Almost certainly this is not the case, and the structures and their incorporated materials should be considered as anisotropic and viscoelastic. Further, the complexity of the materials and structures exists on several scales pertinent to the mechanical function (as a millimeter wide structure, micrometers thick vibrates nanometers), and is always capable of providing new surprises such as the liquid cavities in the locust tympanal membrane, or the lipid in the weta ear. The question for researchers in the future will continue to be how to work with all these confounding issues such that any computer modeling and simulation provides useful additions to our knowledge. 
The second point, and the end to this chapter, is to remember that these mechanical specializations found in the insect ears are inextricably linked to the evolutionary pressures acting on the different species. So while it can be very interesting for engineers, physicists, and mathematicians to look at how an ear functions, seeking understanding of the physical principles that are being utilized, it is important to ask 'why?'. The hearing organ performs a function relating to the ecology of the insect, therefore a full understanding of its function is only possible by connecting how it works with why it should do so, as discussed in the other chapters in this volume.

\section{References}

Bennet-Clark, H. C. (1998). Size and scale effects as constraints in insect sound communication. Philosophical Transactions of the Royal Society B: Biological Sciences, 353, 407-419.

Bies, D.A., \& Hansen, C.H. (2009). Engineering Noise Control: Theory and Practice. New York, NY: Taylor \& Francis Ltd.

Boo, K.S., \& Richards, A.G. (1975). Fine structure of the scolopidia in the Johnston's organ of male Aedes aegypti (L.) (Diptera: Culicidae), International Journal of Insect Morphology and Embryology, 4, 549-566.

Camalet, S., Duke, T., Jülicher, F., \& Prost, J. (2000). Auditory sensitivity provided by selftuned critical oscillations of hair cells. Proceedings of the National Academy of Sciences of the USA, 97, 3183-3188.

Clements, A.N. (1999). The Biology of Mosquitoes: Sensory reception and behavior. Oxford, UK: Chapman \& Hall.

Coro, F., Kössl, M. (1998). Distortion-product otoacoustic emissions from the tympanic organ in two noctuoid moths. Journal of Comparative Physiology A, 183, 525-531. 
Eguíluz, V.M., Ospeck, M., Choe, Y., Hudspeth, A.J., \& Magnasco, M.O. (2000). Essential Nonlinearities in Hearing. Physics Review Letters, 84, 5232-5235.

Field, L.H., Hill, K.G., \& Ball, E.E. (1980). Physiological and Biophysical Properties of the Auditory-System of the New-Zealand Weta Hemideina-Crassidens (Blanchard, 1851) (Ensifera, Stenopelmatidae). Journal of Comparative Physiology, 141, 31-37.

Fletcher, N.H. (1992). Acoustic Systems in Biology. New York, NY: Oxford University Press. Fonseca, P.J., Munch, D., \& Hennig, R.M. (2000). Auditory perception - How cicadas interpret acoustic signals. Nature, 405, 297-298.

Göpfert, M. C., Humphris, A. D. L., Albert, J. T., Robert, D., \& Hendrich, O. (2005). Power gain exhibited by motile mechanosensory neurons in Drosophila ears. Proceedings of the National Academy of Sciences of the USA, 102, 325-330.

Göpfert, M.C., \& Robert, D. (2001). Active auditory mechanics in mosquitoes. Proceedings of the Royal Society B, 268, 333-339.

Göpfert, M.C., \& Robert, D. (2003). Motion generation by Drosophila mechanosensory neurons. Proceedings of the National Academy of Sciences of the USA, 100, 5514-5519. Gordon, S.D, Jackson, J.C., Rogers, S.M., \& Windmill, J.F.C. (2014). Listening to the environment: hearing differences from an epigenetic effect in solitarious and gregarious locusts. Proceedings of the Royal Society B: Biological Sciences, 281, 20141693.

Gordon, S.D., \& Windmill, J.F.C. (2015). Hearing ability decreases in ageing locusts. Journal of Experimental Biology, 218, 1990-1994.

Jackson, J.C., \& Robert, D. (2006). Nonlinear auditory mechanism enhances female sounds for male mosquitoes. Proceedings of the National Academy of Sciences of the USA, 103, 16734-16739.

Kemp, D.T. (1978). Stimulated acoustic emissions from within the human auditory system. Journal of the Acoustical Society of America. 64, 1386-1391. 
Kössl, M. \& Boyan, G.S. (1998). Acoustic distortion products from the ear of a grasshopper. Journal of the Acoustical Society of America, 104, 326-335.

Lomas, K.F., Greenwood, D.R., Windmill, J.F.C., Jackson, J.C., Corfield, J. \& Parsons, S. (2012). Discovery of a Lipid Synthesising Organ in the Auditory System of an Insect. PLOS One, 7, No. e51486.

Malkin, R., McDonagh, T.R., Mhatre, N., Scott, T.S., \& Robert, D. (2014). Energy localization and frequency analysis in the locust ear. Journal of the Royal Society Interface, 11, No. 20130857.

Manley, G.A., Fay, R.R., \& Popper, A.N. (Eds.) (2008). Active Processes and Otoacoustic Emissions in Hearing. New York, NY: Springer-Verlag.

Mhatre, N., \& Robert, D. (2013). A tympanal insect ear exploits a critical oscillator for active amplification and tuning. Current Biology, 23, 1952-1957.

Michelsen, A. (1971). Physiology of Locust Ear: 1-3. Zeitschrift für vergleichende Physiologie, 71, 49-128.

Miles, R.N., Robert, D., \& Hoy, R.R. (1995). Mechanically coupled ears for directional hearing in the parasitoid fly Ormia ochracea. Journal of the Acoustical Society of America, 98, 3059-3070.

Miller, L. A. (1970). Structure of green lacewing tympanal organ (Chrysopa carnea, Neuroptera). Journal of Morphology, 131, 359-382.

Moir, H.M., Jackson, J.C., \& Windmill, J.F.C. (2013). Extremely high frequency sensitivity in a 'simple' ear. Biology Letters, 9, No. 20130241.

Montealegre-Z, F., Jonsson, T., Robson-Brown, K.A., Postles, M., \& Robert, D. (2012).

Convergent evolution between insect and mammalian audition. Science, 338, 968-971. Montealegre-Z, F., \& Robert, D. (2015). Biomechanics of hearing in katydids. Journal of Comparative Physiology A, 201, 5-18. 
Mora, E.C., Cobo-Cuan, A., Macías-Escrivá, F., \& Kössl, M. (2015). Unexpected dynamic up-tuning of auditory organs in day-flying moths. Journal of Comparative Physiology A: Neuroethology, Sensory, Neural, and Behavioral Physiology, 201, 657-666.

Palghat Udayashankar, A., Kössl, M., \& Nowotny, M. (2012). Tonotopically arranged traveling waves in the miniature hearing organ of bushcrickets. PLOS One, 7, e31008. Pikovsky, A., \& Kurths, J. (2003). Synchronization: A Universal Concept in Nonlinear Sciences. Cambridge, UK: Cambridge University Press.

Reddy, J.N. (2005). An Introduction to the Finite Element Method, $3^{\text {rd }}$ Edition. New York, NY: McGraw-Hill Education.

Robles, L., \& Ruggero, M. A. (2001). Mechanics of the mammalian cochlea. Physiological Reviews, 81, 1305-1352.

Roeder, K.D., \& Treat, A.E. (1957). Ultrasonic Reception by the tympanic organ of noctuid moths. Journal of Experimental Zoology, 134, 127-157.

Stephen, R.O., \& Bennet-Clark, C. (1982). The anatomical and mechanical basis of stimulation and frequency analysis in the locust ear. Journal of Experimental Biology, 99, 279-314.

Sueur, J., Janique, S., Simonis, C., Windmill, J.F.C., \& Baylac, M. (2010). Cicada ear geometry: species and sex effects. Biological Journal of the Linnean Society, 101, 922-934. Surlykke, A., Filskov, M., Fullard, J.H., \& Forrest, E. (1999). Auditory relationships to size in noctuid moths: bigger is better. Naturwissenschaften, 86, 238-241.

Todi, S.V., Sivan-Loukianova, E., Jacobs, J.S., Kiehart, D.P., \& Eberl, D.F. (2008). Myosin VIIA, important for human auditory function, is necessary for Drosophila auditory organ development. PLOS One, 3, No. e2115.

Treat, A.E., \& Roeder, K.D. (1959). A nervous element of unknown function in the tympanic organs of moths. Journal of Insect Physiology, 3, 262-270. 
van Dijk, P., Wit, H., \& Segenhout, J. (1989). Spontaneous optoacoustic emissions in the European edible frog (Rana esculenta): spectral details and temperature dependence. Hearing Research, 42, 273-282.

von Békésy, G. (1960). Experiments in Hearing. New York, NY: McGraw-Hill.

Windmill, J.F.C., Gopfert, M.C., \& Robert, D. (2005). Tympanal traveling waves in migratory locusts. Journal of Experimental Biology, 208, 157-168.

Windmill, J.F.C., Jackson, J.C., Tuck, E.J., \& Robert, D. (2006). Keeping up with Bats:

Dynamic Auditory Tuning in a Moth. Current Biology, 16, 2418-2423.

Windmill1, J.F.C., Sueur, J., \& Robert, D. (2009). The next step in cicada audition:

measuring pico-mechanics in the cicada's ear. Journal of Experimental Biology, 212, 40794083.

Yack, J. E. (2004). The structure and function of auditory chordotonal organs in insects.

Microscopy Research and Technique, 63, 315-337.

Yager, D.D., \& Hoy, R.R. (1986). The Cyclopean Ear - A New Sense for the Praying-Mantis. Science, 231, 727-729. 